\title{
Spring Cold Bias of SST and Minimal Wind Mixing in the Equatorial Pacific Cold Tongue
}

\author{
LIN Peng-Fei, LIU Hai-Long, LI Chao, and ZHANG Xue-Hong \\ State Key Laboratory of Numerical Modeling for Atmospheric Sciences and Geophysical Fluid Dynamics, Institute of Atmospheric \\ Physics, Chinese Academy of Sciences, Beijing 100029, China
}

Received 10 November 2010; revised 12 November 2010; accepted 15 November 2010; published 16 November 2010

\begin{abstract}
The authors investigate the relationship between bias in simulated sea surface temperature (SST) in the equatorial eastern Pacific cold tongue during the boreal spring as simulated by an oceanic general circulation model (OGCM) and minimal wind mixing (MWM) at the surface. The cold bias of simulated SST is the greatest during the boreal spring, at approximately $3^{\circ} \mathrm{C}$. A sensitivity experiment reducing MWM by one order of magnitude greatly alleviates cold biases, especially in MarchApril. The decrease in bias is primarily due to weakened vertical mixing, which preserves heat in the uppermost layer and results in warmer simulated SST. The reduction in vertical mixing also leads to a weak westward current in the upper layer, which further contributes to SST warming. These findings imply that there are large uncertainties about simple model parameters such as MWM at the oceanic surface.
\end{abstract}

Keywords: cold biases, the equatorial Pacific cold tongue, the minimal wind mixing

Citation: Lin, P.-F., H.-L. Liu, C. Li, et al., 2010: Spring cold bias of SST and minimal wind mixing in the equatorial Pacific cold tongue, Atmos. Oceanic Sci. Lett., 3, 342-346.

\section{Introduction}

In most oceanic general circulation models (OGCMs), simulated sea surface temperatures (SSTs) are too cold and extend too far westward when compared against observed data (e.g., Stockdale et al., 1993; Griffies et al., 2009). The problem also exists in most no-flux adjusted coupled models (e.g., Mechoso et al., 1995). Researchers have investigated many aspects of this problem, including the effect of ocean color distribution (e.g., Murtugudde et al., 2002), the effect of oceanic current on wind stress (Luo et al., 2005), the advection scheme (Xiao and Yu, 2006), and wave-induced mixing (Song et al., 2007). Although each of these factors can be adjusted to alleviate the cold bias to some extent, cold bias is still a common problem in climatic GCMs (Griffies et al., 2009).

In OGCMs, which are used in climate system models and to study climate problems, a constant minimal threshold-value of surface mixing due to wind, hereafter called the minimum wind mixing (MWM), is employed to mimic the turbulent mixing due to high-frequency wind and to keep SST from becoming too warm (Pacanowski, 1995; Li et al., 2001). This coefficient is always assigned

Corresponding author: LIU Hai-Long, lhl@lasg.iap.ac.cn to a constant value (approximately $10 \mathrm{~cm}^{2} \mathrm{~s}^{-1}$ ) in climate models. In reality, however, this value is not a constant, and the magnitudes of wind stresses vary considerably in space and time. For example, the surface wind is much larger in the eastern equatorial Pacific than in the western equatorial Pacific. Moreover, the trade winds in the eastern equatorial Pacific relax during the boreal spring. Furthermore, close investigations of SST biases indicate that the largest cold biases occur in the boreal spring (Fig. 1a), when the surface mixing is supposed to be small. In this study, we investigate the possibility that overestimated surface wind mixing in models contributes to a cold bias in SST during the boreal spring.

We begin by evaluating simulated SST biases in an OGCM in the equatorial eastern Pacific. The relationship between these cold biases and the artificial surface vertical mixing in the eastern equatorial Pacific is explored through a sensitivity experiment. The paper is organized as follows: the model and sensitivity experiments are introduced in the following section, Section 3 shows the results and the last section consists of a brief summary of our findings.

\section{Model description, experiments, and observational datasets}

In this study, we use the LASG/IAP (State Key Laboratory of Numerical Modeling for Atmospheric Sciences and Geophysical Fluid Dynamics/Institute of Atmospheric Physics) Climate System Ocean Model (LICOM, Zhang et al., 2003; Liu et al., 2004a, b). The LICOM model has a horizontal resolution of $0.5^{\circ} \times 0.5^{\circ}$ and has 30 vertical layers, with 12 regular layers ( 25 meters per layer) above $300 \mathrm{~m}$ and 18 irregular layers below $300 \mathrm{~m}$. The model is driven by climatology wind stresses and heat fluxes from Röske (2001), which are derived from the European Center for Medium-Range Weather Forecast (ECMWF) 15-year reanalysis (ERA-15) (Gibson et al., 1997). The model SST and sea surface salinity (SSS) are restored to SST and SSS from World Ocean Atlas 98 (WOA98, http://www.nodc.noaa.gov/), respectively.

In LICOM, the vertical mixing scheme follows that of Pacanowski and Philander (1981, hereafter PP). The scheme can be written as follows.

$$
v=\frac{v_{0}}{(1+5 R i)^{2}}+v_{\mathrm{b}},
$$




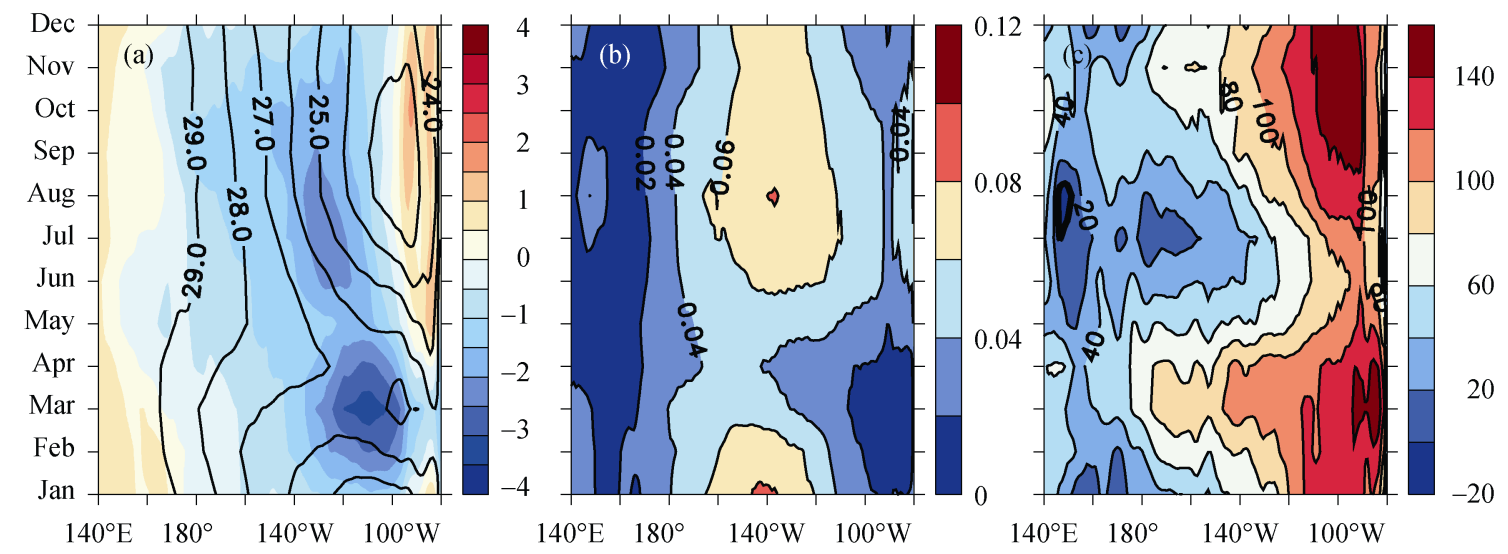

Figure 1 (a) Climatologically monthly SST biases (CTL minus Reynolds SST, filled) and Reynolds SST (contour, $1^{\circ} \mathrm{C}$ interval) at the equator (averaged from $2^{\circ} \mathrm{S}-2^{\circ} \mathrm{N}$ ); Climatologically monthly (b) wind stress magnitudes (Pa) and (c) net heat fluxes (W $\mathrm{m}^{-2}$, positive is downward) used for driving the OGCM along the equator.

$$
\begin{gathered}
\kappa=\frac{v}{(1+5 R i)}+\kappa_{\mathrm{b}}, \\
R i=\frac{g}{\rho_{0}} \frac{\partial \rho}{\partial z} /\left[\left(\frac{\partial U}{\partial z}\right)^{2}+\left(\frac{\partial V}{\partial z}\right)^{2}\right],
\end{gathered}
$$

where $v$ and $\kappa$ are the viscosity and diffusivity coefficients, respectively. The viscosity $v_{0}$ equals $50 \mathrm{~cm}^{2} \mathrm{~s}^{-1}$. The background viscosity and diffusivity coefficients are represented by $v_{\mathrm{b}}$ and $\kappa_{\mathrm{b}}$, their respective values are $1 \mathrm{~cm}^{2}$ $\mathrm{s}^{-1}$ and $0.1 \mathrm{~cm}^{2} \mathrm{~s}^{-1} . R i$ is the bulk Richardson number and $g$ is gravity acceleration. $\rho$ and $\rho_{0}$ are potential density of sea water, and its reference value $\left(=1029 \mathrm{~kg} \mathrm{~m}^{-3}\right)$, respectively. $U$ and $V$ represent zonal and meridional velocity, respectively.

As mentioned in the introduction, an MWM of $10 \mathrm{~cm}^{2}$ $\mathrm{s}^{-1}$ is used in the top layer of the model. In order to examine the cold biases of SST and the effect of MWM, two experiments are conducted. First, LICOM is run for 950 years starting from zero oceanic velocity. This run is the control run (hereafter CTL). We evaluate the performance of the CTL run against the observed data and calculate the biases in simulated SST. A second, 50-year integration was conducted beginning after the 900th year of CTL. In this run, MWM is reduced by one order of magnitude to $1 \mathrm{~cm}^{2} \mathrm{~s}^{-1}$. This run is referred to as the sensitivity run (hereafter SEN). Results from the last five years of the integration are used for comparison. In the tropical Pacific, the modeled SST will reach a quasi-balance after ten years when LICOM is run forced by climatological winds and heat fluxes. Thus, the result derived from the last five-year integrations cannot affect the conclusion of the study.

SST values from Reynolds and Smith (1994) and oceanic subsurface temperatures from WOA01 (Stephens et al., 2002) are employed to evaluate temperatures simulated using LICOM. In addition, currents from Simple Ocean Data Assimilation Version 2.0.2 (SODA 2.0.2, Carton et al., 2005) are used for comparison with the simulated currents.

\section{Results}

In the equatorial eastern Pacific cold tongue, the observed SST is the warmest in March-April and the coldest in August-September (Fig. 1a). During the March-April period, the relaxed easterly wind (Fig. 1b) leads to a weak upwelling at the equator due to a reduced poleward Ekman flow. This process is essential to warming SST at the equator. The weak wind also causes both a large net surface heat flux (Fig. 1c) due to a reduction in the surface latent heat flux and weak surface vertical mixing due to strong stratification. The weak upwelling, large surface heat flux and weak mixing can all potentially lead to a warm SST anomaly in March-April.

However, in the same period (March-April), simulated SST has the greatest cold bias (approximately $3^{\circ} \mathrm{C}$ ) at approximately $110^{\circ} \mathrm{W}$ (Fig. 2a). At the same time, the simulated mixed layer depth (MLD) is approximately 20 $\mathrm{m}$ deeper than the observed MLD (Fig. 2c). These results suggest an overly strong mixing in March-April in the CTL run. Strong mixing would keep heat from accumulating on the ocean surface and cause colder SST values. In addition to the March-April cold bias, the simulated SST east of $100^{\circ} \mathrm{W}$ is warmer than observed along the South American coast during May-December (Fig. 2a).

To verify the effect of MWM on SST, a sensitivity experiment is conducted with MWM at the surface reduced by one order of magnitude. After this reduction in MWM, the simulated cold bias of SST in the Pacific cold tongue is alleviated significantly, especially in March-April (Fig. 2b). The bias of MLD during March-April (> $20 \mathrm{~m}$, Fig. 3c) is also reduced in the SEN run (Fig. 3d). However, the change in SST is small in other months. These results indicate that the effects of surface MWM on SST and MLD in March-April are considerable.

To understand the relationship between the reduction in MWM and the alleviation of SST cold bias in MarchApril, the differences in both the frequency of use of MWM and the averaged diffusivity are shown first. The positive value of the frequency in Figs. 3a and 3b indicates that the computed diffusivity is smaller than MWM 

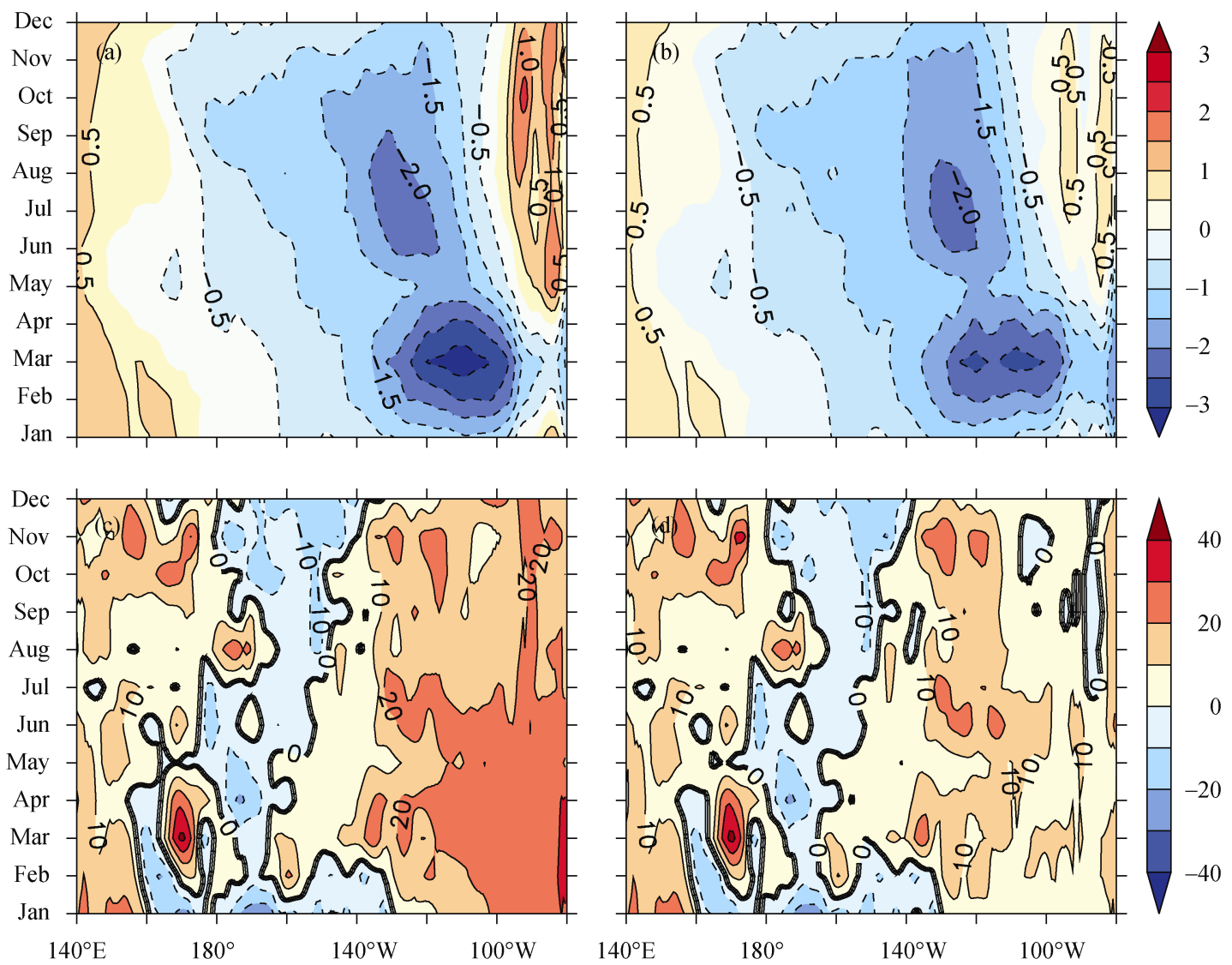

Figure 2 Climatologically monthly SST biases (a) CTL minus Reynolds SST and (b) SEN minus Reynolds; climatologically monthly mixed layer depth (meter) biases (c) CTL minus WOA and (d) SEN minus WOA.
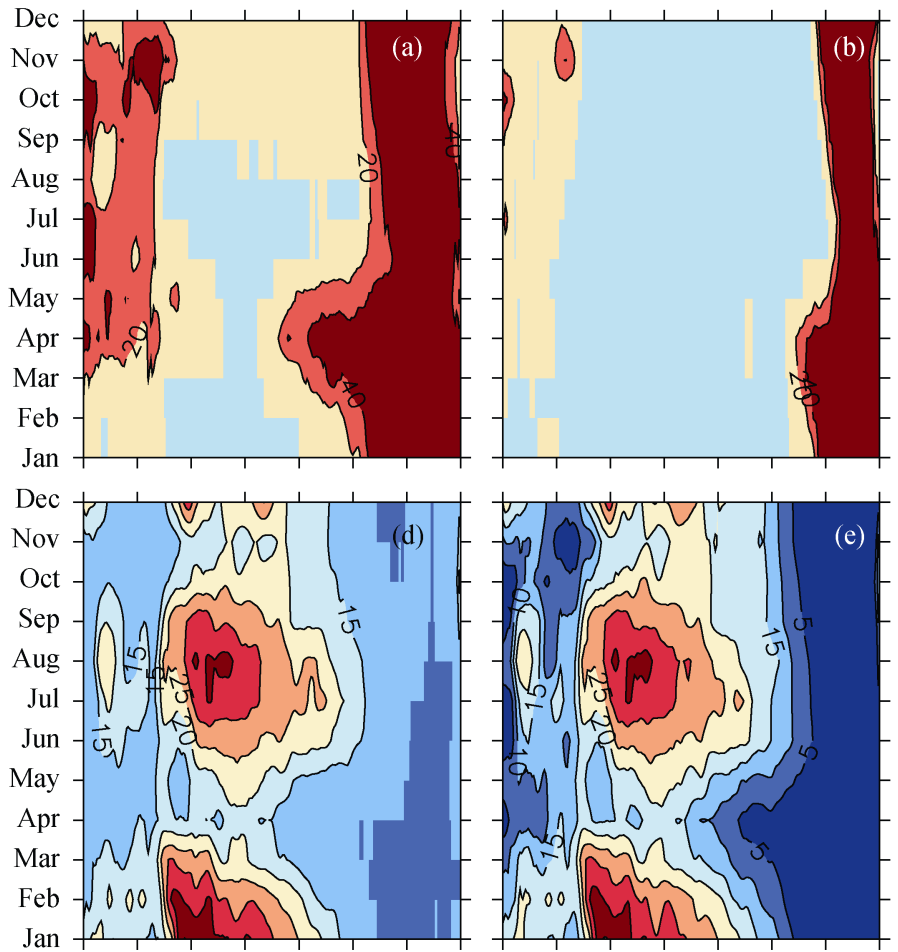

$140^{\circ} \mathrm{E} \quad 180^{\circ} \quad 140^{\circ} \mathrm{W} \quad 100^{\circ} \mathrm{W} \quad 140^{\circ} \mathrm{E}$

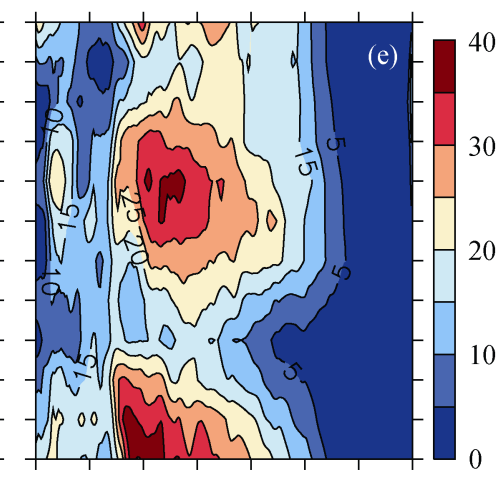

$140^{\circ} \mathrm{E} \quad 180^{\circ} \quad 140^{\circ} \mathrm{W} \quad 100^{\circ} \mathrm{W}$
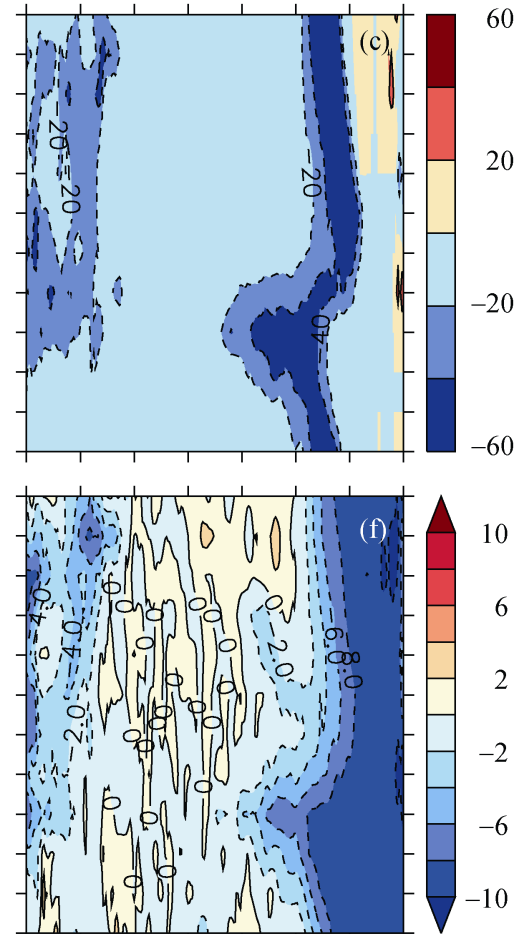

Figure 3 The frequency (\%) of use of minimal wind mixing for (a) CTL, (b) SEN, and (c) their difference (SEN minus CTL). The diffusivity used in the actual simulation $\left(\mathrm{cm}^{2} \mathrm{~s}^{-1}\right)$ for (d) CTL, (e) SEN, and (f) their difference (SEN minus CTL). 
during one month. In these periods or regions, MWM will be substituted for the computed diffusivity, leading to a high diffusivity. For the CTL run, the highest frequency of used MWM (beyond 40\%) primarily occurs east of $120^{\circ} \mathrm{W}$. The $40 \%$ contour can extend to $140^{\circ} \mathrm{W}$ in March-April (Fig. 3a). The averaged diffusivity for the CTL run (Fig. 3d) contoured with the $40 \%$ frequency is around $10 \mathrm{~cm}^{2} \mathrm{~s}^{-1}$.

After MWM is reduced at the surface, the $40 \%$ frequency contour shrinks eastward by $20^{\circ}$ (Fig. 3b). The large difference occurs primarily between $100^{\circ} \mathrm{W}$ and $120^{\circ} \mathrm{W}$, where the computed diffusivities are between 1 and $10 \mathrm{~cm}^{2} \mathrm{~s}^{-1}$ (Fig. 3c). The corresponding diffusivities in the SEN run are smaller than in the CTL run in the equatorial eastern Pacific by approximately $9 \mathrm{~cm}^{2} \mathrm{~s}^{-1}$ (Figs. 3e-f). Due to the reduction in surface mixing, the MLD becomes shallow, and upper layer stratification is enhanced in the equatorial eastern Pacific (Fig. 2d). SST then increases as the MLD becomes shallower, especially in March-April.

MWM also affects the simulation of ocean currents. In the boreal spring, the relaxation of the easterly wind also weakens the upwelling and surface westward South Equatorial Current (SEC) (Fig. 4a). Meanwhile, the eastward equatorial undercurrent (EUC) strengthens significantly and uplifts to the surface (Fig. 4a). EUC tends to warm SST in March-April. In the SEN run, the reduced MWM at the surface causes a larger eastward current as the easterly wind relaxes. EUC uplifts to the surface and causes large eastward zonal current anomalies $(>16 \mathrm{~cm}$ $\mathrm{s}^{-1}$ ) near the sea surface between $100-120^{\circ} \mathrm{W}$ (Fig. 4d). The strong eastward EUC anomalies will result in warm advection (e.g., Wang and McPhaden, 1999) and warm simulated SST in March-April.

\section{Summary}

This study investigates the bias of simulated SST in the equatorial eastern Pacific cold tongue in boreal spring and its relationship with MWM at the surface. The cold bias of simulated SST is largest in March, at approximately $3^{\circ} \mathrm{C}$ between $120^{\circ} \mathrm{W}$ and $100^{\circ} \mathrm{W}$. A sensitivity experiment with MWM reduced by one order of magnitude shows an alleviation of cold biases in SST, especially during the boreal spring. According to our analysis, the warming is primarily due to weakened vertical mixing, which preserves more heat in the uppermost layer. The reduction of vertical mixing also leads to a weak westward current in the upper layer, which further contributes to the warming.

These results indicate that the cold biases in the eastern equatorial Pacific may also be partially attributable to sub-grid parameterization. Surface MWM is assigned a value of $10 \mathrm{~cm}^{2} \mathrm{~s}^{-1}$ in most OGCMs, which is much too large for the cold tongue. Our findings imply that there are large uncertainties about some simple parameters like MWM. The application of these parameters needs to be re-evaluated for configuration of the present model.

Acknowledgements. The study is jointly supported by the National Basic Research Program of China (Grant Nos. 2010CB950502, 2010CB951904, and 2010AA012303), LASG Free Exploration Fund, and the National Natural Science Foundation of China (Grant Nos. 40906012 and 40775054).
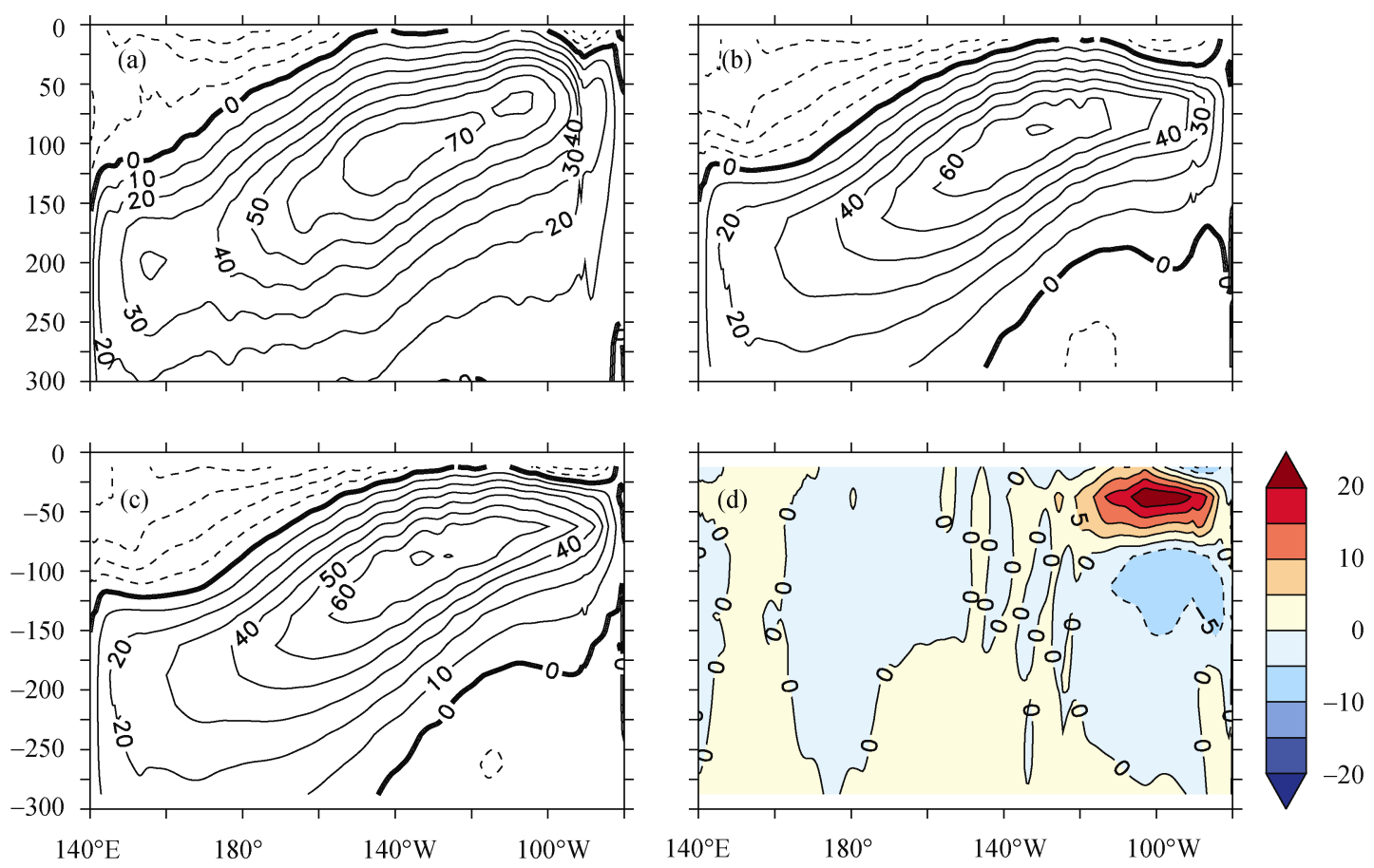

Figure 4 The climatologically mean zonal ocean current $\left(\mathrm{cm} \mathrm{s}^{-1}\right)$ in April averaged from $2^{\circ} \mathrm{S}-2^{\circ} \mathrm{N}$ for (a) SODA, (b) CTL, (c) SEN, and (d) the difference of averaged zonal ocean current between SEN and CTL (SEN minus CTL). 


\section{References}

Carton, J. A., B. S. Giese, and S. A. Grodsky, 2005: Sea level rise and the warming of the oceans in the Simple Ocean Data Assimilation (SODA) ocean reanalysis, J. Geophys. Res., 110 C09006, doi:10.1029/2004JC002817.

Gibson, J. K., P. Kållberg, S. Uppala, et al., 1997: ERA description, in: ECMWF ERA-15 Project Report Series, No. 1, European Centre for Medium-Range Weather Forecasts, Shinfield, Reading, 72pp.

Griffies, S. M., A. Biastoch, C. Böning, et al., 2009: Coordinated Ocean-ice Reference Experiments (COREs), Ocean modell., 26, $1-46$.

Li, X.-J., Y. Chao, J. C. McWilliams, et al., 2001: A comparison of two vertical-mixing schemes in a Pacific ocean general circulation model, J. Climate, 14, 1377-1398.

Liu, H.-L., Y.-Q. Yu, W. Li, et al., 2004a: Manual for LASG/IAP Climate System Ocean Model (LICOM1.0) (in Chinese), Science Press, Beijing, 1-28.

Liu, H.-L., X.-H. Zhang, W. Li, et al., 2004b: A eddy-permitting oceanic general circulation model and its preliminary evaluations, Adv. Atmos. Sci., 21, 675-690.

Luo, J.-J., S. Masson, E. Roeckner, et al., 2005: Reducing climatology bias in an ocean-atmosphere CGCM with improved coupling physics, J. Climate, 18, 2344-2360.

Mechoso, C. R., A. W. Robertson, N. Barth, et al., 1995: The seasonal cycle over the tropical Pacific in coupled ocean-atmosphere general circulation models, Mon. Wea. Rev., 123, 2825-2838.

Murtugudde, R., J. Beauchamp, C. R. McClain, et al., 2002: Effects of penetrative radiation on the upper tropical ocean circulation, $J$ Climate, 15, 470-486.

Pacanowski, R. C., 1995: MOM 2 Documentation, User's Guide and Reference Manual, GFDL Ocean Group Tech. Rep. 3, 232pp.

Pacanowski, R. C., and S. G. H. Philander, 1981: Parameterization of vertical mixing in numerical models of tropical oceans, $J$. Phys. Oceanogr., 11, 1443-1451.

Reynolds, R. W., and T. M. Smith, 1994: Improved global sea surface temperature analyses using optimum interpolation, J. Climate, 7, 929-948.

Röske, F., 2001: An Atlas of Surface Fluxes Based on the ECMWF Re-Analysis-A Climatological Dataset to Force Global Ocean General Circulation Models, Report No. 323, Max-PlanckInstitute für Meteor., Hamburg, 31pp.

Song, Z.-Y., F.-L. Qiao, Y.-Z. Yang, et al., 2007: An improvement of the too cold tongue in the tropical Pacific with the development of an ocean-wave-atmosphere coupled numerical model, Prog. Nat. Sci., 17(5), 576-583.

Stephens, C., J. I. Antonov, T. P. Boyer, et al., 2002: World Ocean Atlas 2001, Volume 1: Temperature, NOAA Atlas, NESDIS 49, U.S. Government Printing Office, Washington D.C., 176pp.

Stockdale, T., D. Anderson, M. Davey, et al., 1993: Intercomparison of Tropical Ocean GCMs, World Climate Research Programme, WRPC-79, WMO/TD-No. 545, 43pp.

Wang, W., and M. J. McPhaden, 1999: The surface-layer heat balance in the equatorial Pacific ocean. Part I: Mean seasonal cycle, J. Phys. Oceanogr., 29, 1812-1831.

Xiao, C., and Y. Q. Yu, 2006: Adoption of a two-step-shape-preserving advection scheme in an OGCM, Prog. Nat. Sci. (in Chinese), 16(11), 1442-1448.

Zhang, X.-H., Y.-Q. Yu, and H.-L. Liu, 2003: The development and application of the oceanic general circulation models, Part I. The Global Oceanic General Circulation Models, Chinese J. Atmos. Sci. (in Chinese), 27(4), 607-617. 